\title{
Unusual Drug Fever Caused by Imipenem/Cilastatin and a Review of Literature
}

\author{
Jie Yang, MD, Qing Wang, PhD, Suyu Wang, MD, Yufeng Zhang, PhD, Zhinong Wang, PhD
}

Department of Cardiothoracic Surgery, Changzheng Hospital, Second Military Medical University, Shanghai, China

\section{ABSTRACT}

Introduction: Drug fever is a febrile reaction caused by initiation of one drug or varieties of drugs and often disappears after cessation of the $\operatorname{drug}(\mathrm{s})$. Clinically, drug fever is frequently induced by antibiotics, anticonvulsants, and antineoplastics. There are few previous reports about drug fever caused by imipenem/cilastatin.

Case Presentation: Here, we described a 66-year-old man undergoing the Ivor Lewis esophagectomy for esophageal carcinoma, who developed drug fever. The patient had a high temperature with shivering after administration of imipenem/cilastatin for 7 days. Furthermore, his temperature came down after discontinuing imipenem/cilastatin and receiving steroids. Body temperature increased rapidly 4 hours after intravenous readministration of imipenem/cilastatin and rapidly decreased to normal after discontinuing imipenem/cilastatin and administering steroids.

Conclusion: Thorough history, blood tests, physical examination, and computed tomography (CT) did not reveal any evidence of fever. Drug fever caused by imipenem/cilastatin was considered. We also present a review of relevant literature and provide a point of reference for the clinical diagnosis and therapy of patients with drug fever.

\section{INTRODUCTION}

Drug fever (DF) is a febrile reaction caused by initiation of one drug or varieties of drugs and often disappears after cessation of the drug(s). DF is mostly induced by antibiotics, anticonvulsants, antineoplastics, immunoregulators, and other cardiac agents [Cunha 2007; Patel 2010; Vodovar 2012; Cunha 2013]. Amikacin, ofloxacin, rifampin, vancomycin, and sulfamethoxazole are relatively more frequent to induce DF

Received August 4, 2018; received in revised form November 19, 2018; accepted November 22, 2018.

Abbreviations: CRP, C-reactive protein; CT, computed tomography; ESR, erythrocyte sedimentation; MRI, magnetic resonance imaging; $q 8 \mathrm{~h}$, every 8 hours; q12h, every 12 bours; QD, quaque die ("every day"); WBC, white blood cell.

Correspondence: Zhinong Wang, Department of Cardiothoracic Surgery, Changzheng Hospital, Second Military Medical University, Shanghai, China 200003; +86-(0)21-881885905; fax: +86-(0)21-81885901 (e-mail: wangzn007@smmu.edu.cn).
[Vodovar 2012]. The possibility of DF should be considered in cases of unexplained fever without clear medical evidence of infection [Vodovar 2012]. We describe a case of DF caused by imipenem/cilastatin (Tienam), review relevant literature, and summarize the characteristics of DF. Informed consent was obtained from the patient for publication of the report.

\section{CASE DESCRIPTION}

On October 17, 2017, a 66-year-old man was admitted to the Department of Cardiothoracic Surgery, Changzheng Hospital, because of progressive dysphagia for 1 month. He had hypertension for the past 10 years, but his blood pressure was well controlled by oral valsartan. A definitive diagnosis of esophageal cancer was confirmed after careful physical examination, gastroscopy, and chest computed tomography (CT). On October 26, 2017, the patient was treated with the Ivor Lewis esophagectomy. The surgical technique was described simply as the following: after general anesthesia, the patient was placed supine with the right shoulder upward 30 degrees. After ligating and dissecting the azygos vein, the esophagus was resected through a right fourth intercostal anterolateral incision. Gastric tube and intestinal feeding tube insertions were completed through an upper midline abdominal incision. Then, the gastric tube was delivered into

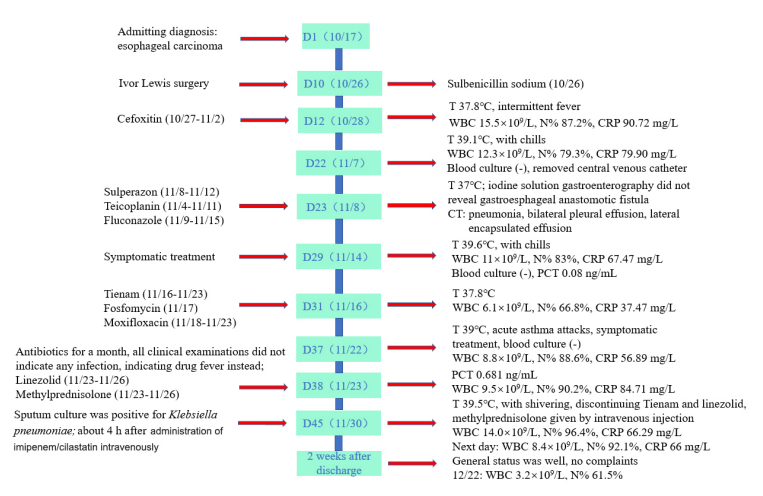

Figure 1. The detailed treatment process. “D1 (10/17)” and similar wording follow this type of meaning: "day 1 (October 17)." Likewise, items such as "(10/26)" and "(10/27-11/2)" follow this type of meaning: "(October 26)" and "(October 27-November 2)." T, temperature; N\%, neutrophils' percentage; (-), negative; PCT, procalcitonin. 


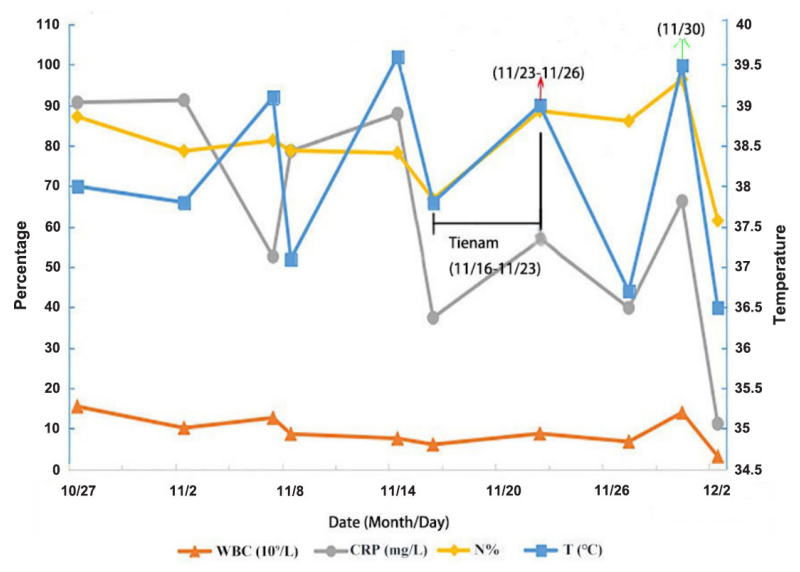

Figure 2. Trend of some important indices. Red arrow means methylprednisolone administration. Green arrow indicates 4 hours after administration of Tienam with temperature of $39.5^{\circ} \mathrm{C}$ and chills, so Tienam was discontinued and methylprednisolone injected. T, temperature; N\%, neutrophils' percentage.

the right thorax, and a circular stapled end-to-side esophagogastric anastomosis was fashioned in the upper mediastinum. Total lymphadenectomy was performed including lymph nodes along the bilateral recurrent nerves and those resected during standard lymphadenectomy. Seven days after surgery, he was still febrile, with a fluctuating temperature between $37^{\circ} \mathrm{C}$ and $37.8^{\circ} \mathrm{C}$. Thus, piperacillin/tazobactam combined with teicoplanin was administered. At $7 \mathrm{PM}$ on November 7,2017 , he developed intense shivering with fever $\left(39.1^{\circ} \mathrm{C}\right)$, and the symptoms became progressively severe. The temperature returned to normal after the central venous catheter was removed. On November 8, sputum culture was positive for Klebsiella pneumoniae. Then, sulperazone and teicoplanin combined with fluconazole were administered according to the results. His temperature was normal until 1:30 PM on November 14 , when he had an acute febrile attack $\left(39.6^{\circ} \mathrm{C}\right)$ accompanied by chills. After administration of chlorpheniramine and continuous physical cooling, his temperature decreased. On November 16, we adjusted the anti-infection therapy to $1 \mathrm{~g}$ imipenem/cilastatin $\mathrm{q} 8 \mathrm{~h}+2 \mathrm{~g}$ fosfomycin q12h. On November 18, we added $0.4 \mathrm{~g}$ moxifloxacin QD with imipenem/cilastatin owing to an adverse reaction to fosfomycin. He had a temperature of $39^{\circ} \mathrm{C}$ and an acute asthma attack on November 22. Finally, noninvasive ventilation was provided to improve ventilation, with promethazine injection. Finally, his symptoms improved and his temperature gradually lowered.

Because other examination did not indicate local infection, systemic infection, rheumatic disease, or other reasons for the fever, we considered DF. On November 23, with discontinuation of imipenem/cilastatin and moxifloxacin, adding $40 \mathrm{mg}$ methylprednisolone QD for 3 days to control inflammation, we wanted to clarify whether it was DF or not by observing the symptoms, considering the general status, and conducting auxiliary examination. Linezolid

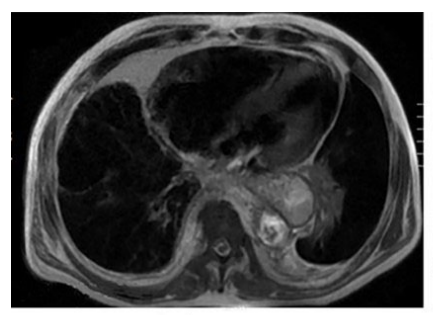

A

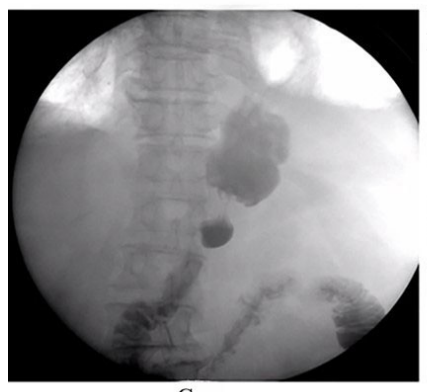

C

Figure 3. Imaging tests: (A), MRI; (B), CT; (C), gastrointestinal iodine contrast; (D), x-ray.

was administered simultaneously because a sputum culture tested positive for Enterococcus faecium. His temperature was normal and all inflammatory markers were normal. On November 30, $1 \mathrm{~g}$ intravenous imipenem/cilastatin was administered according to the sputum culture results of $K$. pneumoniae that was sensitive to imipenem/cilastatin. After 4 hours, his temperature reached $38^{\circ} \mathrm{C}$ with shivering. Furthermore, his signs and symptoms rapidly progressed, and the temperature reached $39.5^{\circ} \mathrm{C}$. So, we discontinued imipenem/cilastatin and linezolid. The patient recovered and had no fever for 2 weeks after his discharge. The detailed treatment process is shown in Figure 1. The important indices are shown in Figure 2.

\section{DISCUSSION}

Despite its frequent occurrence, DF is frequently ignored and misdiagnosed by clinicians [Fang 2016]. Most DF develops 7-10 days after drug administration, and temperature returns to normal after drug discontinuation within 48 hours to 72 hours [Patel 2010; Cunha 2013]. Although the mechanisms of DF are still unknown, the most likely theories include hypersensitivity and immune-mediated reactions [Johnson 1996]. Other mechanisms include antibody-antigen complexes, drug effects on thermoregulation, and idiosyncratic reactions [Linares 2011]. Based on previous reports, the diagnostic criteria for DF include the following [Young 1982; Pleasants 1994]: (1) a temperature above $37.5^{\circ} \mathrm{C}$; (2) no signs of infection after detailed examinations; (3) return of the temperature to normal after drug discontinuation within 48 hours to 72 hours; (4) rapid increases in body temperature after administration of the drug (namely the drug provocation test (DPT) is positive); (5) exclusion of other possible causes of fever. It is still difficult to diagnose DF through the 
Previous Case Reports of Drug Fever Caused by Antibiotics*

\begin{tabular}{|c|c|c|c|c|c|}
\hline Tigecycline & Shao [Shao 2015] & 2015 & Male/62 & Pneumonia & $\begin{array}{l}21 \text { days later; } \mathrm{T} 39^{\circ} \mathrm{C} \text {; with rash; WBC, ESR and } \\
\mathrm{CRP} \text { were elevated; other examination did not } \\
\text { indicate any infection; general status was well; } \\
\text { temperature reduced after discontinuation of } \\
\text { tigecycline and use of DEX } 2 \mathrm{mg} \text { QD for } 3 \text { days; } \\
\text { inflammatory indices were normal in } 3 \text { days }\end{array}$ \\
\hline Doxycycline & Yuan [Yuan 2016] & 2016 & Female/48 & Brucellosis & $\begin{array}{l}7 \text { days later; } \mathrm{T} 38^{\circ} \mathrm{C} \text {; WBC was normal; ESR and } \\
\mathrm{CRP} \text { were moderately elevated; other examina- } \\
\text { tion could not explain the reason; after doxycy- } \\
\text { cline discontinuation, temperature was reduced to } \\
\text { normothermia after } 24 \text { hours }\end{array}$ \\
\hline Piperacillin/tazobactam & Park [Park 2011] & 2011 & Female/72 & Infectious arthritis & $\begin{array}{l}2 \text { weeks later; } \mathrm{T} 39^{\circ} \mathrm{C} \text {; with rash; eosinophil count } \\
\text { and CRP were mildly elevated; other tests were } \\
\text { negative; after discontinuing the drug, tempera- } \\
\text { ture returned to normal; DPT was positive }\end{array}$ \\
\hline Piperacillin/tazobactam & Li [Li 2015] & 2015 & Female/36 & Incision infection & $\begin{array}{c}3 \text { days later; } \mathrm{T} 39^{\circ} \mathrm{C} \text {; WBC, ESR and eosinophil } \\
\text { count were elevated; other tests revealed no } \\
\text { evidence of fever; the temperature returned } \\
\text { to normal } 4 \text { days after piperacillin/tazobactam } \\
\text { cessation }\end{array}$ \\
\hline Piperacillin/tazobactam & Linares [Linares 2011] & 2011 & Male/65 & Tendon infection & $\begin{array}{c}13 \text { days later; } \mathrm{T} 39^{\circ} \mathrm{C} \text {; with shivering, rash, pruri- } \\
\text { tus; the temperature returned to normal } 48 \text { hours } \\
\text { after the drug discontinuation; } 4 \text { days later, rash } \\
\text { disappeared; DPT was positive }\end{array}$ \\
\hline Teicoplanin & Ochi [Ochi 2011] & 2011 & Male/38 & $\begin{array}{l}\text { MRSA infection on } \\
\text { the LVAS }\end{array}$ & $\begin{array}{l}9 \text { days later; } \mathrm{T} 38^{\circ} \mathrm{C} \text {; WBC was dropped; CRP } \\
\text { was mildly elevated; other tests did not find signs } \\
\text { of the fever; with a decrease in plasma concen- } \\
\text { tration of teicoplanin, temperature returned to } \\
\text { normal gradually after teicoplanin cessation }\end{array}$ \\
\hline
\end{tabular}

*T, temperature; DEX, dexamethasone; LVAS, left ventricular assist system.

aforementioned diagnostic criteria, especially when it overlaps with the course of anti-infection therapy [Li 2015]. We have summarized some previous DF reports (Table). DF may be accompanied with chills, rash, pruritus, and other symptoms besides typical febrile characteristics. The percentage of neutrophils, CRP, and ESR can increase simultaneously. Because of the previous infection, the diagnosis of DF should be deliberative. The diagnosis is established after infection is ruled out. DF should be considered if medical examinations do not reveal the cause of fever, such as a history of previous infections and increments of WBC, CRP, and ESR. The diagnosis of DF is based on exclusion [Park 2011; Li 2015]. If necessary, DPT should be performed after the patients have been informed of adverse effects that may occur during the test.
In spite of previous infection and inflammation, the patient recovered after the antibiotic treatment was adopted according to the sputum culture and auxiliary tests. CT, MRI, and gastrointestinal iodine contrast were used many times, but they did not find apparent causes of local or systemic infection (Figure 3). T-SPOT®.TB (Oxford Diagnostic Laboratories, Memphis, TN, USA) and immunological test results were negative. A multidisciplinary discussion led to the possibility of DF; this possibility conformed with the exclusion criteria. In addition, chills, high temperature, and acute asthma were observed after administration of imipenem/cilastatin for 7 days. However, blood culture, routine blood test, and other indices were negative. The symptoms and temperature were relieved 
after discontinuing imipenem/cilastatin and administering methylprednisolone for 3 days.

Although the first sputum culture was sensitive $K$. pneumoniae, the patient did not have fever after discontinuation of antibiotics. When $K$. pneumoniae was cultured for the second time, the patient had no discomfort and fever, although fever occurred after administration of Tienam and temperature decreased after discontinuation of the drug. If his fever were caused by $K$. pneumoniae, the patient's symptoms would not improve, and fever would still be present after drug cessation. However, in our case, temperature returned to normal after drug cessation, and we injected $40 \mathrm{mg}$ methylprednisolone. This was consistent with DPT results, which confirmed DF caused by imipenem/cilastatin. The patient never had any fever since discharge. Therefore, we believe the fever was caused by imipenem/ cilastatin, instead of $K$. pneumoniae.

Imipenem/cilastatin was the first carbapenem antibiotic approved by the FDA. The most common side effects include rash, diarrhea, nausea, vomiting, liver and kidney damage, and epilepsy, but fever is rare [Hornik 2013]. It has been reported that imipenem/cilastatin can cause psychiatric symptoms [Ninan 2016], eosinophilic pneumonia [Foong 2016] and white cell aplasia [Kalambokis 2005].

To the best of our knowledge, there are few reports of DF induced by imipenem/cilastatin. The body is more sensitive to the drug in elderly patients who have taken different kinds of antibiotics [Shao 2015]. Hypersensitivity is considered to be a type III allergic reaction [Ochi 2011]. Before administration of imipenem/cilastatin, our patient received cefoxitin, piperacillin/tazobactam, sulperazone, teicoplanin, and fluconazole, all of which can produce some allergic factors and make him susceptible to DF." Our patient had a high temperature with shivering after administration of imipenem/cilastatin for 7 days. Furthermore, his temperature came down after discontinuing imipenem/cilastatin and receiving steroids. Body temperature increased rapidly 4 hours after intravenous readministration of imipenem/cilastatin and rapidly decreased to normal after discontinuing imipenem/cilastatin and administering steroids. This is in line with the standard of DPT. Fever occurred rapidly after drug use again and was relieved after drug discontinuation within 48 hours to 72 hours. In addition, all inflammatory marker tests and imaging examinations did not show obvious infection. Therefore, our case is highly suspected to be DF caused by imipenem/cilastatin. Because of the short duration of Avelox and fosfomycin, DF induced by them may not be considered. In addition, because no fever developed with linezolid monotherapy, DF induced by linezolid was not taken into consideration, either.

In cases of DF, WBC, ESR, and CRP may be increased in different degrees. Moreover, rash, pruritus, and shivering may be seen in DF [Linares 2011]. In spite of that, especially because of DF overlapping with the duration of anti-infection therapy, DF should not be ignored by clinicians. Monitoring these inflammatory markers can help clinicians to confirm DF [Patel 2010; Yuan 2016]. When DF is suspected by clinicians, the drug should be discontinued. If necessary, a low dose of the drug can be administered to confirm the diagnosis. It is necessary to inform patients of the risk and prepare a first aid drug before taking DPT. A low-dose steroid can facilitate inflammation recession, especially when the adverse reaction is severe and fatal [Shao 2015].

Our patient developed DF caused by imipenem/cilastatin. Although DF is not a serious complication, it may lead to unnecessary costs, prolonged hospitalization, and overuse of antibiotics, which results in the generation of drug-resistant bacteria [Vodovar 2012; Yuan 2016]. Though the diagnosis of DF is challenging, in cases of unexplained fever, especially in the absence of apparent infection, the diagnosis of DF should be considered.

\section{ACKNOWLEDGMENTS}

Written consent was obtained from the patient.

\section{REFERENCES}

Cunha BA. 2007. Fever of unknown origin: clinical overview of classic and current concepts. Infect Dis Clin North Am 21(4):867-915.

Cunha BA. 2013. Clinical approach to fever in the neurosurgical intensive care unit: Focus on drug fever. Surg Neurol Int 4(suppl 5):S318-22.

Fang Y, Xiao H, Tang S, Liang L, Sha W, Fang Y. 2016. Clinical features and treatment of drug fever caused by anti-tuberculosis drugs. Clin Respir J 10(4):449-54.

Foong KS, Lee A, Pekez M, Bin W. 2016. Imipenem/cilastatin-induced acute eosinophilic pneumonia. BMJ Case Rep 2016:bcr2016214804.

Hornik CP, Herring AH, Benjamin DK Jr, et al. 2013. Adverse events associated with meropenem versus imipenem/cilastatin therapy in a large retrospective cohort of hospitalized infants. Pediatr Infect Dis J 32(7):748-53.

Johnson DH, Cunha BA. 1996. Drug fever. Infect Dis Clin North Am 10(1):85-91.

Kalambokis G, Vassou A, Bourantas K, Tsianos EV. 2005. Imipenemcilastatin induced pure white cell aplasia. Scand J Infect Dis 37(8):619-20.

Li Z, Shen J, Li Q, Chan MT, Wu WK. 2015. Drug fever induced by piperacillin/tazobactam in a scoliosis patient: a case report. Medicine (Baltimore) 94(46):e1875.

Linares T, Fernández A, Soto MT, Escudero E, Gacías L. 2011. Drug fever caused by piperacillin-tazobactam. J Investig Allergol Clin Immunol 21(3):250-1.

Naschitz JE. 2011. Drug fever induced by ertapenem. QJM 104(8):730-1.

Ninan J, George GM. 2016. Imipenem-cilastatin-induced psychosis: a case report. J Med Case Rep 10:107.

Ochi H, Wada K, Okada H, et al. 2011. The persistence of druginduced fever by teicoplanin--a case report. Int J Clin Pharmacol Ther 49(5):339-43.

Park HB, Choi JS, Park SH, Kee WJ, Koh YI. 2011. Drug fever due to piperacillin/tazobactam loaded into bone cement. J Korean Med Sci 26(2):301-3

Patel RA, Gallagher JC. 2010. Drug fever. Pharmacotherapy 30(1):57-69. 
Pleasants RA, Walker TR, Samuelson WM. 1994. Allergic reactions to parenteral beta-lactam antibiotics in patients with cystic fibrosis. Chest 106(4):1124-8.

Shao QQ, Qin L, Ruan GR, Chen RX, Luan ZJ, Ma XJ. 2015. Tigecycline-induced drug fever and leukemoid reaction: a case report. Medicine (Baltimore) 94(45):e1869.

Vodovar D, LeBeller C, Mégarbane B, Lillo-Le-Louet A, Hanslik T.
2012. Drug Fever: a descriptive cohort study from the French national pharmacovigilance database. Drug Saf 35(9):759-67.

Young EJ, Fainstein V, Musher DM. 1982. Drug-induced fever: cases seen in the evaluation of unexplained fever in a general hospital population. Rev Infect Dis 4(1):69-77.

Yuan HL, Lu NW, Xie H, Zheng YY, Wang QH. 2016. Doxycyclineinduced drug fever: a case report. Infect Dis (Lond) 48(11-12):844-6. 